Cahiers de recherches médiévales

\title{
Héthoum de Korykos historien arménien
}

Un prince cosmopolite à l'aube du XIV ${ }^{\mathrm{e}}$ siècle

\section{Claude Mutafian}

\section{(2) OpenEdition Journals}

Édition électronique

URL : https://journals.openedition.org/crm/2526

DOI : $10.4000 / \mathrm{crm} .2526$

ISSN : 1955-2424

Éditeur

Honoré Champion

Édition imprimée

Date de publication : 15 janvier 1996

Pagination : 157-176

ISSN : 1272-9752

Référence électronique

Claude Mutafian, «Héthoum de Korykos historien arménien », Cahiers de recherches médiévales [En ligne], 1 | 1996, mis en ligne le 07 février 2008, consulté le 15 décembre 2022. URL : http:// journals.openedition.org/crm/2526; DOI : https://doi.org/10.4000/crm.2526 


\section{Héthoum de Korykos historien arménien Un prince cosmopolite à l'aube du XIV $\mathrm{X}^{\mathfrak{e}}$ siècle}

Dans le cinquième et demier livre, d'ailleurs posthume, de Rabelais, Pantagruel visite le pays de Satin et rencontre plusieurs modernes historiens. La liste comporte les noms des principaux voyageurs, historiens et géographes connus à l'époque; après Hérodote, Pline, Strabo, ..., entre Jacques Cartier et Marc Paule Venitien (Marco Polo), on lit Charton Armenian'. Il s'agit là du prince arménien Héthoum de Korykos, appelé Ayton ou Hayton en Europe, auteur de «La Fleur des Histoires de la terre d'Orient». Écrite en français en 1307, copiée aussitôt en latin, traduite ensuite du latin au français, l'œuvre eut au Moyen Age un énorme succès. C'est ainsi que, à peine quarante ans plus tard, le chroniqueur florentin Giovanni Villani conseille, pour qui veut en savoir plus sur les «Tartares», de lire le traité de frère Aiton d'Arménie et le Livre du Million de Venise ${ }^{2}$, c'est-à-dire Marco Polo.

Plus d'une cinquantaine de copies manuscrites de «La Fleur des Histoires» nous sont parvenues, présentant l'une des trois versions, en général dans des recueils de récits de voyages médiévaux et souvent en compagnie, entre autres, de Marco Polo: les deux sont à peu près contemporains et offrent à l'Europe des tableaux pittoresques de ces Mongols qui effayaient mais aussi fascinaient. La plupart de ces recueils sont sompteusement enluminés, le plus célèbre étant le Ms. Fr. 2810 de la Bibliothèque nationale de France, connu sous le nom de «Livre des Merveilles»". Dès les débuts de l'imprimerie, les éditions et traductions en diverses langues se succédèrent. L'œuvre de Héthoum, au-delà de sa description in vivo des Mongols, est aussi une précieuse source historique sur cette période qui suivit la fin de la Syrie franque et sur laquelle on a peu de témoignages contemporains. Peu à peu, pourtant, l'auteur sombra dans l'oubli, jusqu'à ce qu'en 1906 le second tome des «Documents arméniens» du «Recueil des historiens des Croisades» publie les deux textes, français et latin, avec une savante introduction de Charles Kohler, récapitulant tout ce dont on disposait à l'époque sur la vie et l'œuvre du prince arménien".

\section{L'Orient chrétien à la fin du XIII' siècle}

La découverte de nouvelles sources, essentiellement arméniennes, rend nécessaire une mise à jour de l'activité de cet étonnant personnage: écrivain et

\footnotetext{
${ }^{1}$ Rabelais, éd. P. Jourda, Paris, Garnier, 1962, t. II, p. 401.

${ }^{2}$ Croniche de Giovanni, Matteo e Filippo Villani, Trieste, 1857, t. I, p. 71, 182.

${ }^{3}$ Louis de Backer, L'Extrême-Orient au Moyen Age, Paris, Leroux, 1877, pp. 125-255.

${ }^{4}$ Recueil des Historiens des Croisades, Documents arméniens, Paris, Imprimerie nationale, 1906, t. II, pp. XXIII-CXLII, 113-253, 255-363. Dans la suite, Hayton, op. cit.
} 
voyageur, guerrier arménien et moine latin, diplomate et comploteur, apôtre d'une nouvelle croisade, témoin cosmopolite essentiel de cette période charnière entre la chute d'Acre (1291) et la fin de l'alliance christiano-mongole (ca 1320), qui a marqué le triomphe, difficile mais définitif, de l'islam au Proche-Orient. Ce fut aussi une période critique pour le Royaume arménien de Cilicie, fondé en 1198 par le prince roupénide Léon dans l'angle nord-est de la Méditerranée. À Léon I' succéda son gendre Héthoum $I^{e r}$ en 1226 , puis, en 1270 , Léon II, fils du précédent : trois grands souverains qui affermirent un puissant royaume, organisé sur le modèle féodal occidental importé par les Francs au Levant. Depuis le milieu du XIII siècle, sa diplomatie était basée sur l'alliance avec les Mongols de Perse face au monde musulman représenté par les Turcs seljoukides d'Anatolie et surtout par les Mamlouks, maîtres de l'Égypte et de la Syrie non littorale. À la mort de Léon II (1289), ceux-ci avaient déjà à plusieurs reprises profité des troubles qu'avaient à affronter les Mongols sur leurs autres frontières pour envahir la Cilicie, et en 1285 Léon II avait dû concéder un traité de paix.

Son fils Héthoum II prenait le pouvoir dans un contexte difficile, d'autant plus que la chute $d^{\prime}$ Acre laissait bientôt, comme seul État chrétien au Levant autre que la Cilicie, le Royaume latin de Chypre, depuis près d'un siècle aux mains de la dynastie poitevine des Lusignan, alliée aux Héthoumides arméniens par de nombreux mariages. L'année suivante (1292) les Mamlouks attaquèrent la forteresse de Hromgla, sur l'Euphrate, siège du patriarche suprême arménien, appelé «catholicos» - ayant refusé le concile de Chalcédoine (451), l'Église arménienne était autocéphale depuis le $\mathrm{VI}^{e}$ siècle. La prise de Hromgla obligea le catholicossat à se déplacer à Sis, capitale du royaume de plus en plus menacé. Héthoum II n'était pas l'homme de la situation: ses penchants monacaux le faisaient alterner des périodes de règne (d'ailleurs sans couronne) et des séjours au couvent, laissant alors le trône à tel ou tel de ses frères avec le risque de voir le «remplaçant» prendre goût au pouvoir.

\section{Korykos et ses seigneurs}

La fin des États latins de Syrie donna un coup de fouet au déjà florissant port cilicien d'Ayas (Lajazzo en italien), alors seul point d'échange entre l'Occident et l'Extrême-Orient non musulman. Cette importance d'Ayas est née avec le royaume, supplantant le port «historique» de Korykos, plus à l'ouest, où l'on peut encore voir les restes imposants des deux forteresses, celle de la terre et celle de la mer ${ }^{5}$. Mais Korykos restait un fief convoité, et durant tout le XII ${ }^{e}$ siècle il fut disputé entre les Byzantins, les Normands d'Antioche et la dynastie arménienne des Roupénides. On ne connaît pas de nom de seigneur de Korykos durant cette période. Le Theobaldus de Corizo ou Corith mentionné dans les Regestes de Jérusalem ${ }^{6}$ entre 1134 et 1151 est attribué par erreur à Korykos, il s'agit de Cyrthus en Syrie. Par contre, dans la

\footnotetext{
${ }^{5}$ F. Hild \& H. Hellenkemper, Kilikien und Isaurien, Vienne (Tabula Imperii byzantini 5), 1990, t. I, pp. 315-320.

R.W. Edwards, The fortifications of Armenian Cilicia, Washington D.C. (Dumbarton Oaks Studies 23), 1987, pp. 161-167.

${ }^{6}$ R. Röhricht, Regesta regni Hierosolymitani, Innsbrück, 1893, p. 37, 67.
} 
liste des barons présents au couronnement de Léon $I^{\text {er }}$ le 6 janvier 1198 figure un Simon prince de Korykos ${ }^{7}$. À cette époque le port semble être fermement attaché aux domaines arméniens.

Un peu plus tard, en 1210, on trouve sur un chrysobulle adressé par Léon I ${ }^{\text {er }}$ aux Hospitaliers une liste de témoins, parmi lesquels Baharam, filius Gofredi de $\mathrm{Corco}^{8}$, c'est-à-dire "Vahram, fils de Godefroy seigneur de Korykos». On doit donc en déduire qu'après Simon la seigneurie était passée à un certain Godefroy, noble franc d'après son nom, ce qui correspondait bien à la politique du roi. Godefroy a dû s'«arméniser», peut-être par mariage, car son fils porte un nom arménien. On retrouve ce Vahram sur un autre chrysobulle ${ }^{9}$ aux Hospitaliers (1214) et sur le second privilège ${ }^{10}$ octroyé par Léon $I^{\pi}$ aux Génois (1215). Il avait alors le grade de «maréchal», seconde dignité après celle de «connétable» (général en chef des armées du royaume). Sur le premier document il est suivi du nom de son frère Jocelyn (un nom franc), mais sur le second il est explicitement cité comme seigneur de Korykos. On peut donc supposer qu'il succéda à son père en 1215 dans ce fief. Il n'allait le conserver que cinq ans. En effet, après la mort de Léon $I^{\text {er }}$, il participa à la rébellion de Raymond-Roupen, petit-neveu du défunt roi et un moment prévu pour lui succéder, après avoir été imposé comme prince d'Antioche. Or Léon avait finalement opté pour sa fille Zabel. Raymond-Roupen, voulant s'emparer du trône par force, débarqua à Korykos en 1220 et enrôla sous sa bannière plusieurs princes arméniens dont le maréchal Vahram de Korykos, qui épousa sa mère Alice (nièce de Léon $\mathrm{I}^{\mathrm{er}}$ ). Les rebelles furent combattus par le régent Constantin de Baberon et les barons loyalistes. Après quelques succès, comme les prises de Tarse et Adana, ils furent capturés et les meneurs, dont Vahram, mis à mort ${ }^{11}$.

Le fief de Korykos fut alors conquis par le régent, qui voulut le donner à l'un de ses fils, Ochine. Mais l'aîné, le connétable Sempad, fit valoir ses droits, et Constantin dut consulter son gendre Jean d'lbelin, légiste réputé, qui déclara valable la décision ${ }^{12}$ : le fief ayant été conquis et non hérité, son possesseur n'est pas astreint à respecter le droit d'aînesse. Ochine, frère du roi Héthoum $\mathrm{I}^{\mathrm{er}}$, reçut donc, au cours du second quart du XIII' siècle, le fief de Korykos, qui passait ainsi à une branche collatérale de la famille royale. Il est qualifié en 1264 de seigneur de la magnifique forteresse de Korykos, de Mitizon, de Manion, de Gantchi et d'autres châteaux

\footnotetext{
'Sempad le Connétable, Chronique, en arménien, Moscou, 1856, p. 101.

Chronique du royaume de la Petite Arménie, éd. et trad. Dulaurier, Paris, Imprimerie nationale (Recueil des Historiens des Croisades, Documents arméniens I), 1869, p. 638.

La chronique attribuée au connétable Smbat, trad. Gérard Dédéyan, Paris, Paul Geuthner

(Documents relatifs à l'histoire des Croisades XIII), 1980, p. 78.

${ }^{8}$ Röhricht, op. cit., p. 225.

Victor Langlois, Le trésor des chartes d'Arménie, Venise, 1863, p. 116.

${ }^{9}$ Langlois, op. cit., p. 213.

${ }^{10}$ Röhricht, op. cit., p. 236.

Langlois, op. cit., p. 126.

"Bar Hebraeus, Chronique, trad. anglaise Wallis Budge, Londres, 1932, p. 380.

Chroniques mineures, éd. V. Hakobian, en arménien, Erevan, 1951, t. I, p. 79, 1956, t. II, p. 64, 104.

Michel le Syrien, Chronique, en arménien, Jérusaleme, 1871, pp. 515-6.

${ }^{12}$ Rec. Hist. Croisades, Lois, Paris, 1841, t. I, p. 220.
} 


\section{Claude MUTAFIAN}

moins importants ${ }^{13}$. Une inscription sur le château de Baberon ${ }^{14}$ nous apprend qu'il était aussi bailli du royaume, c'est-à-dire régent. En 1262, il avait été envoyé auprès du légat du pape, à Acre, comme ambassadeur pour discuter des divergences entre les Églises ${ }^{15}$. Un peu plus tôt, en mars 1255 , le missionnaire franciscain Roubrouck, passant à Sis au retour de Mongolie, rencontra Constantin père du roi qui attendait le retour de son fils Héthoum $\mathrm{I}^{\text {er }}$ parti lui aussi auprès du khan. Roubrouck vit tous ses fils, sauf un, appelé Barunusin (baron Ochine), qui faisait construire une forteresse $^{16}$ - il s'agissait probablement de travaux à Korykos.

\section{Héthoum, seigneur de Korykos}

Ochine de Korykos mourut en $1265^{17}$, mais son fief allait alors rester dans la famille, sur laquelle des détails sont donnés par l'historien arménien Tchamtchian (1786), basés sur des sources qui ne nous sont pas connues ${ }^{18}$. Selon lui, les trois fils d'Ochine de Korykos, appelés Ochine, Grégoire et Héthoum (c'est notre historien) par ordre d'âge, étaient neveux du maréchal Ochine. Cet Ochine descendait de la branche aînée des Héthoumides, celle des seigneurs de Lampron, il était l'oncle de la reine Guérane, épouse de Léon II ; la Pierpont Morgan Library de New York possède un superbe évangéliaire de 1274 commandé par le maréchal Ochine et comportant un important colophon ${ }^{19}$.

Toujours selon Tchamtchian, le fils aîné Ochine hérita du fief de Gantchi et fut nommé sénéchal en 1277, puis connétable plus tard, probablement vers la fin du siècle. Cette dignité suprême était en effet passée, à la mort de Sempad frère du roi

\footnotetext{
${ }^{13}$ La chronique attribuée..., op. cit., p. 115.

${ }^{14}$ Léonce Alishan, Sissouan, trad. Sirounian et Issaverdens, Venise, Saint-Lazare, 1899, p. 77.

${ }^{15}$ Réc. Hist. Croisades, Doc. arm, op. cit., t. I, p. 692.

${ }^{16}$ A. van den Wyngaer, Sinica Franciscana, Quaracchi, 1929, t. I, p. 329.

Guillaume de Rubrouck, Voyage dans l'empire mongol, trad. C. et R. Kappler, Paris, Payot, 1985, p. 243.

${ }^{17}$ La chronique attribuée..., op. cit., p. 116.
}

Le bréviaire du roi Ochine, éd. Surmélian, en arménien, Antilias, 1933, p. 99.

Sempad, op. cit., p. 115.

Chroniques mineures, op. cit., t. I, p. 83, t. II, p. 73.

Rec. Hist. Croisades, Doc. arm, op. cit., t. I, p. 651.

${ }^{18}$ M. Tchamtchian, Histoire d'Arménie, en arménien, Venise, Saint-Lazare, 1786, t. III, p. 279.

Alishan, op. cit., p. 401.

${ }^{19} \mathrm{D}$. Balian, «Un ancien évangéliaire à Césarée», en arménien, Handes Amsorya, 12, 1898 , pp. 244-248.

F. Macler, «Quelques feuillets épars d'un tétraévangile arménien», Revue des études arméniennes, 6, 1926, pp. 169-176.

Treasures in heaven, ed. Mathews et Wieck, New York, The Pierpont Morgan Library, 1994, pp. 193-194.

A. Matévossian, Colophons de manuscrits arméniens (XIIle siècle), en arménien, Erevan, 1984, p. 439. 
Héthoum $\mathrm{I}^{\mathrm{er}}$, en 1275 , à son fils Léon de Baberon ${ }^{20}$, qui la portait encore en 1289 , lorsque le pape Nicolas IV lui écrivit ${ }^{21}$ à l'occasion du passage en Cilicie du franciscain Monte Corvino. Le second fils, Grégoire, devint bailli (régent) du royaume et reçut le fief de Korykos ${ }^{22}$, mais il mourut peu après 1277 , et son frère cadet, Héthoum, devint prince de Korykos vers 1280 . Il était donc cousin germain à la fois du roi Léon II (par son père) et de la reine Guérane (par sa mère). La filiation paternelle est confirmée par l'intéressé sur le colophon ${ }^{23}$ d'un évangéliaire copié pour lui à Korykos en 1293. Il se nomme Héthoum fils d'Ochine frère du roi Héthoum d'Arménie et seigneur de Korykos, de Manion, de Bounar, d'Anamour entre autres. De plus, dans son «Histoire des Tatares», il explique qu'il parle comme celui qui fut présent en personne $e^{24}$ seulement à partir du khanat d'Abagha (1265), se basant, pour l'époque antérieure, sur la tradition orale jusqu'à l'avènement de Mongka (1251) et, entre 1251 et 1265, sur les récits de son oncle roi Haïton ${ }^{25}$.

Ce passage nous permet aussi de placer autour de 1240 sa date de naissance, par ailleurs inconnue. Il nous laisse aussi supposer que, avant de recevoir, vers 1280 , le fief de Korykos, il avait vécu une partie de son enfance à Sis, à la cour de son oncle mort en 1270. Les «Lignages d'Outre-Mer» nous renseignent sur son mariage avec une princesse de la famille des Ibelin. On y lit en effet que Guy, fils de Baudouin d'Ibelin, épousa Marie, fille du roi Haïton d'Arménie (...); ils eurent un fils et une fille, Thoros et Isabelle (...). Isabelle épousa Haïton, le sire du Courc (seigneur de Korykos) et ils eurent quatre fils et une fille ${ }^{26}$. On a la confirmation du mariage entre la fille de Héthoum $I^{\text {ta }}$ et le fils de Baudouin d'Ibelin dans la dispense papale $^{27}$ du 17 mars 1266, nécessaire pour raisons de consanguinité. Le mariage entre Héthoum de Korykos et Isabelle d'Ibelin était d'ailleurs lui aussi consanguin puisque le roi Héthoum Itr était à la fois oncle paternel de l'époux et grand-père maternel de l'épouse. On ne connaît pas la date du mariage, mais, dans la chronique d'Amadi, l'héritier du fief, donc le fils aîné de ce mariage, est qualifié en 1310 de jeune seigneur de Korykos ${ }^{28}$, ce qui laisse supposer pour ce mariage une date comprise entre 1280 et 1290. Deux colophons de 1293 mentionnent l'épouse Zabel du prince ${ }^{29}$.

\footnotetext{
${ }^{20}$ Sempad, op. cit., p. 130.
}

Rec. Hist. Croisades, Doc. arm. op. cit., t. I, p. 680.

${ }^{21}$ Acta Romanorum Pontificum ab Innocentio $V$ ad Benedictum XI, éd. Delorme et Tautu, Vatican, 1954, p. 157.

${ }^{22}$ Alishan, op. cit., p. 401.

Matévossian, op. cit., p. 516.

${ }^{23}$ Collection privée.

${ }^{24}$ Hayton, op. cit., p. 213.

${ }^{25}$ Ibid.

${ }^{26}$ Rec. Hist. Croisades, Lois, Paris, 1843, t. II, p. 449.

W. Rudt de Collenberg, «Les Ibelin aux XIII ${ }^{e}$ et XIV siècles», Epeteris, Nicosie, 9, 1977 1979, p. 157, 169.

${ }^{27}$ Acta Urbani IV, Clementis IV, Gregorii X, éd. Tautu, Vatican, 1953, p. 53.

${ }^{28}$ Amadi, Chronique, éd. René de Mas Latrie, Paris, Imprimerie nationale, 1891, p. 324.

${ }^{29}$ Collection privée (cf. n. 23).

Matévossian, op. cit., p. 719. 
Héthoum de Korykos était manifestement un lettré. On possède un Recueil manuscrit copié en 1287 sur ordre du prince des princes, de sang royal, pieux et aimant Dieu, Héthoum seigneur de Korykos ${ }^{30}$ comme l'indique le colophon, qui donne là un terminus ante quem pour la seigneurie. Un peu plus tard, pour l'an 1293, on a mentionné le colophon d'un évangéliaire commandé par le prince; le scribe et le peintre s'appellent tous deux Basile. L'année suivante, en 1294, Héthoum commandait un Recueil médical. Cet important manuscrit ${ }^{31}$ nous est parvenu, il est connu sous le nom de «Traité Gagik-Héthoumian» car il s'ouvre sur une copie, la plus ancienne conservée, de la traduction arménienne d'un traité du médecin arabe Abou Ali Sina (Avicenne), une traduction effectuée à la fin du $X^{c}$ siècle sous le roi Gagik I ${ }^{\text {er }}$ d'Ani et qui figure d'ailleurs dans d'autres manuscrits ${ }^{32}$. Le nom du commanditaire, Héthoum de Korykos, se trouve sur les trois colophons du Recueil ${ }^{33}$.

L'œuvre majeure de Héthoum de Korykos est bien entendu «La Fleur des Histoires de la terre d'Orient», mais d'autres écrits, en arménien ceux-là, nous sont parvenus sous son nom. Dans un Recueil copié en 1608 à Constantinople est inséré un colophon écrit en 1293 à Korykos de la main de notre auteur ${ }^{34}$. La fin est identique au colophon de l'évangéliaire qu'il commanda la même année, mais auparavant il rappelle les tristes événements de l'époque, en particulier la chute du siège catholicossal de Hromgla.

\section{Les deux «Chroniques de Héthoum seigneur de Korykos» (1296)}

C'est en 1842 que parut la traduction arménienne de «La Fleur des Histoires», effectuée à Venise par M. Avkérian sur le texte latin. Le traducteur publia, à la fin, une chronologie écrite en 1296 par «Héthoum seigneur de Korykos», basée sur deux manuscrits conservés au couvent mékhitariste de Venise ${ }^{35}$. Le texte fut repris et traduit en français par Dulaurier ${ }^{36}$. On connaît à l'heure actuelle plus d'une dizaine de copies de l'œuvre, et les rédactions peuvent se classer en trois groupes ${ }^{37}$.

${ }^{30}$ Codex Vaticanus Armenus 3, fol. $326^{\mathrm{r}}$.

E. Tisserant, Codices armeni Bybliothecae Vaticanae, Rome, 1927, p. 221.

Matévossian, op. cit., p. 610;

Alishan, op. cit., p. 402.

${ }^{31}$ Bibl. couvent mékhitariste de Venise, Ms. 1281.

K. Zarphanalian, Les anciennes traductions arméniennes, en arménien, Venise, Saint-Lazare, 1889, pp. 761-764.

V. Torkomian, «Un manuscrit médical arménien», en arménien, Bazmavep, 1923, pp. 7-11, 39-43, 69-71.

32 Matenadaran (Bibli. des manuscrits anciens d'Erevan) Ms. 8382.

«Capolavori del Matenadaran», Incontro con il popolo dell'Ararat, Venise, Saint-Lazare, 1987, p. 61.

${ }^{33}$ Alishan, op. cit., p. 402.

${ }^{34}$ Matévossian, op. cit., p. 718.

${ }^{35}$ Héthoum historien des Tatares, éd. M. Avkérian, en arménien, Venise, Saint-Lazare, 2 éd., 1951, pp. 81-90.

${ }^{36}$ Rec. Hist. Croisades, Doc. arm, op. cit., t. I, pp. 469-490.

${ }^{37}$ N. Aguinian, «Compte-rendu», Handès Amsorya, 1957, pp. 505-507. 
Jusqu'au début du siècle, on ne connaissait que la troisième rédaction, réalisée par Nersès Balients; ce dominicain arménien fanatique de l'Union avec la papauté y apporta certaines modifications qui sautent aux yeux si on compare avec les manuscrits du premier groupe. Ceux-ci donnent des informations sur la famille de l'auteur, qui ne laissent aucun doute sur son identification: le texte est écrit non pas par Héthoum de Korykos, comme le dit le titre, mais par le roi Héthoum $\Pi^{38}$. L'édition "authentique» de cette chronique, qui couvre les années 1076-1296, a été réalisée en 1951 par V. Hakobian ${ }^{39}$. Le titre précise que le chroniqueur a utilisé des sources franques, grecques et syriaques. Auparavant, A. Surmélian avait publié en 1933 une description détaillée du «Bréviaire du roi Ochine», un célèbre manuscrit conservé à Alep, copié en 1319 et comportant un exemplaire du premier groupe ${ }^{40}$. Un autre, incomplet (il s'arrête en 1270), figure dans un Recueil conservé à la British Library ${ }^{41}$. Le titre en est singulier, il donne comme auteur Jean serviteur de Dieu. Or le roi Héthoum II s'était fait franciscain justement sous le nom de «frère Jean», ce qui donne un argument de plus pour l'attribution. Pourquoi les autres copies donnent-elles «Héthoum seigneur de Korykos» comme auteur, même celle d'Alep pourtant réalisée en 1319, donc presque contemporaine? L'explication de cette erreur est délicate, et on est bien obligé d'admettre que la confusion entre les deux homonymes s'est transmise d'un scribe à l'autre.

L'affaire se complique avec l'apparition d'un nouvel élément. L'édition de Samuel d'Ani, publiée en 1893 à Etchmiadzine par A. Ter-Mikélian, est basée sur plusieurs copies de ce texte, dont le manuscrit actuellement catalogué $n^{\circ} 1898$ au Matenadaran d'Erevan. Le Recueil s'ouvre sur Samuel d'Ani, après quoi vient une chronique intitulée: "Chronique historique que l'humble serviteur du Christ Héthoum seigneur de Korykos a traduite du français en l'an 745 de l'ère arménienne» $(1296)^{42}$. Voici donc une seconde chronique, écrite la même année et portant en titre le nom du même auteur! Celle-ci ne nous est parvenue que par cette unique copie, elle commence à la naissance du Christ, se termine en 1294, et, selon le titre, n'utilise que des sources franques - essentiellement Martin le Polonais. L'auteur, à côté de ces sources qu'il a adaptées plus que traduites, a inséré de nombreux épisodes de l'histoire arménienne. La première édition a vu le jour en 1956 par les soins de V. Hakobian ${ }^{43}$, qui a d'ailleurs montré que la chronique de Héthoum II s'en est inspirée - beaucoup de passages sont semblables ${ }^{44}$. Mais cette fois rien dans le texte ne permet de mettre en doute l'attribution à Héthoum de Korykos qui figure dans le titre.

\footnotetext{
${ }^{38}$ Chroniques mineures, op. cit. t. I, pp. 65-73.

${ }^{39}$ Id., pp. 74-101.

${ }^{40}$ Le bréviaire du roi Ochine, op. cit., pp. 93-101.

${ }^{41}$ British Library codex Or. 5458 , ff. $135^{2}$ sqq.

F.C. Conybeare, A catalogue of the Armenian manuscripts in the British Museum, Londres, 1913, pp. 290-292.

${ }^{42}$ Samuel d'Ani, Recueil d'histoires, éd. A. Ter-Mikélian, en arménien, Vagharchapat, 1893, p. VIII.

${ }_{43}$ Chroniques mineures, op. cit., t. II, pp. 37-93.

${ }^{4}$ Chroniques mineures, op. cit., t. I. pp. 70-71.
} 
Cette chronique est suivie de plusieurs textes généalogiques ${ }^{45}:$ les histoires des princes roupénides, des rois de Jérusalem, des rois de Chypre et des princes d'Antioche. Là encore, l'auteur est probablement Héthoum de Korykos. Tout cela correspond bien à la latinophilie du prince, dont la carrière a montré sa proximité avec le monde franc et dont la connaissance de la langue française est prouvée par la rédaction française - même si elle a bénéficié de l'aide de Nicolas Falcon — de «La Fleur des Histoires»,

Il semble donc, pour récapituler, qu'on soit en présence de trois textes: la chronique de Héthoum II, la chronique de Héthoum de Korykos, toutes deux écrites en 1296, et les écrits généalogiques de ce même Héthoum de Korykos.

\section{Héthoum vs. Héthoum (1293-1299)}

Entre l'avènement du roi Héthoum II en 1289 et la campagne syrienne du souverain mongol de Perse, Ghazan, en 1299 contre les Mamlouks, Héthoum de Korykos nous dit peu de choses dans «La Fleur des Histoires»:

* Il assista deux fois à l'élection de l'empereur des Tartares ${ }^{46}$, c'est-à-dire au Conseil (appelé Kouriltaï) qui intronisait le nouveau souverain ${ }^{46}$.

* Il passa deux ans en Europe, apud Vallem Viridem ${ }^{47}$ comme le dit le texte latin, seul à mentionner cet épisode qu'on retrouve, incomplet, dans un manuscrit français unique, mais traduit du latin et endommagé par les flammes ${ }^{48}$; il s'agit probablement d'un pèlerinage à l'abbaye de Vauvert, qui peut être N.-D. de Vauvert, à Paris ou dans le diocèse de Nîmes ${ }^{49}$. À son retour, dit-il, le royaume était dans un tel état qu'il renonça momentanément à ses penchants monastiques pour s'occuper de sa patrie.

Une chose est claire: si Héthoum n'a aucune raison d'être mis en doute lorsqu'il relate les faits, leur interprétation, surtout quand il est mis en cause, est à prendre avec beaucoup de précautions. Sa propre chronique ne dit rien sur lui à cette période, pas plus que la chronique qui porte son nom par erreur, au moins dans les copies disponibles jusqu'au début du siècle. Par contre, d'importants détails sont donnés par les copies plus complètes de cette chronique maintenant attribuée, comme on l'a vu, à Héthoum II. On lit ainsi dans la copie contenue dans le «Bréviaire du roi Ochine» (1319):

1293 - Complot contre le roi Héthoum de la part de princes à la tête desquels étaient Héthoum seigneur de Korykos, son frère Ochine seigneur de Gantchi et bien d'autres princesso.

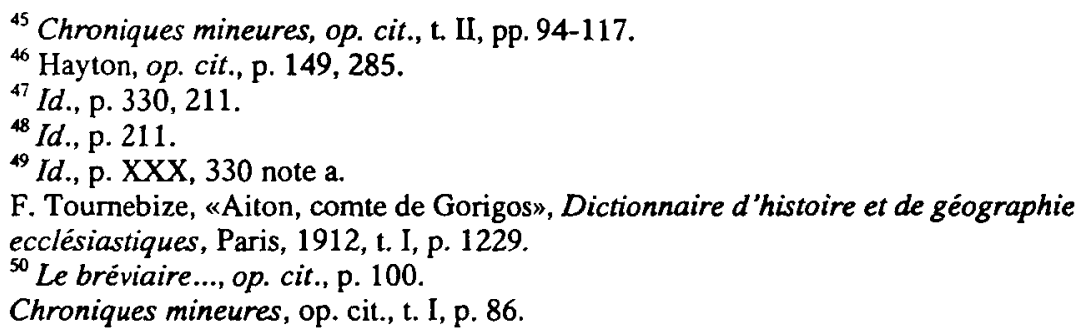




\section{Ochine ${ }^{51}$}

1294 - Fuite de Héthoum seigneur de Korykos et de son frère le seigneur

Or 1293 est l'année où le roi Héthoum fit sa première entrée dans les Ordres, confiant provisoirement la royauté à son frère Thoros avant de la reprendre peu après $^{52}$. Selon cette même chronique du roi lui-même, Thoros reçut de nouveau la royauté en 1296, avant de partir la même année pour Constantinople avec son frère Héthoum $^{53}$, laissant sur le trône un autre frère, Sempad. Entre-temps Héthoum II avait été la cible d'un nouveau complot, dirigé cette fois par Ochine le sénéchal fils du connétable Sempad. La chronique s'arrête là.

À Constantinople, où sa sœur Rita venait d'épouser l'héritier du trône, Michel Paléologue, Héthoum II visita le couvent franciscain ${ }^{54}$, et c'est peut-être là qu'il devint frère mineur sous le nom de Jean - quoique Héthoum de Korykos l'appelle déjà frère Jean au moment du voyage. Après cela, le texte latin seul de «La Fleur des Histoires» nous apprend ${ }^{55}$ - confirmé par les continuations de Samuel d'Ani ${ }^{56}$ et du connétable Sempad ${ }^{57}$ - que le nouveau roi prit goût au pouvoir : au retour de ses deux frères il fit exécuter Thoros et aveugler Héthoum. Un autre frère, Constantin, évinça Sempad en 1297, mais bientôt Héthoum II recouvra la vue et le pouvoir, exilant Constantin et Sempad dans la capitale byzantine et participant peut-être en habit franciscain ${ }^{58}$ - à la campagne de Ghazan en Syrie en 1299.

De tout cela, que pouvons-nous déduire sur la conduite de Héthoum de Korykos dans la décennie précédant la grande guerre mongolo-mamlouke de 1299 ? Attisée par l'attitude ambiguë du roi Héthoum II entre le pouvoir et le couvent et les ambitions de certains de ses frères, l'atmosphère à Sis était propice aux intrigues. Il est donc tout à fait probable qu'en 1293 le seigneur de Korykos ait comploté contre son parent le roi alors au couvent. Au retour de celui-ci, il quitta le pays en 1294. Peut-être est-il allé en Asie mongole, et en ce cas les deux intronisations de khans auxquelles il dit avoir assisté seraient celles de Baïdou, petit-fils de Houlagou, en 1295 , et de son petit cousin Ghazan qui le détrôna la même année. On ne voit d'ailleurs pas de quelles autres il pourrait s'agir - à moins de supposer qu'il ait été présent à l'avènement de Gaikhatou en 1291, ce qui semble bien tôt. Le ton de panégyrique qu'il emploie au sujet de Ghazan, longuement mis en valeur, penche pour notre hypothèse. Peut-être passa-t-il ensuite ses deux années en pèlerinage à Vauvert, en France, ce qui expliquerait en outre sa connaissance de la langue française.

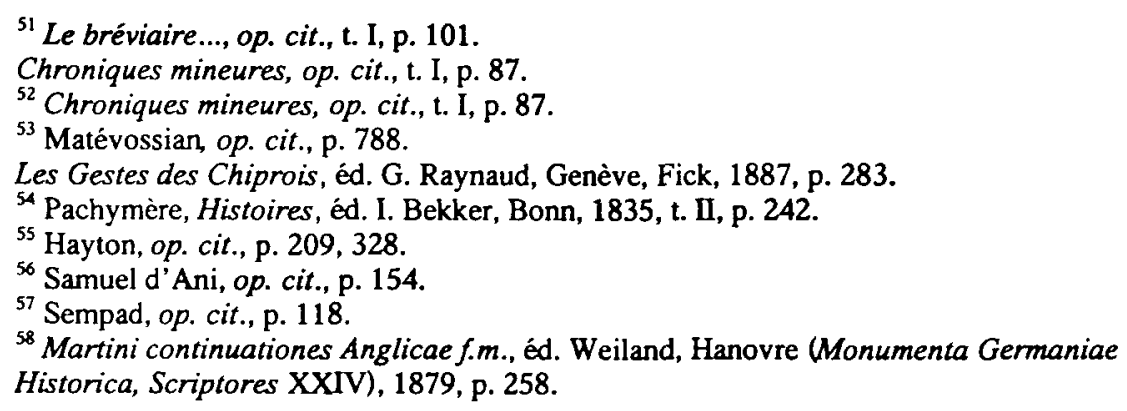


Ceci placerait son retour en Cilicile autour de 1298 , durant le bref gouvernement de Constantin. Les louanges qu'il adresse à ce dernier ${ }^{59}$ dans «La Fleur des Histoires» suggèrent fortement qu'il profita de son arrivée au pouvoir pour revenir d'exil. Le ton est bien différent quand il s'agit de Héthoum II. Certes, les deux colophons de 1293 parlent encore, comme il est de tradition, du seigneur de tous les Arméniens Héthoum, respectueux des lois, vertueux et gloire de l'église ${ }^{60}$ on note le mot «seigneur» et non "roi», à cause de l'entrée au couvent -, mais sur les trois colophons du traité de médecine commandé en 1294 par le seigneur de Korykos, probablement juste au retour de Héthoum II au pouvoir, ce dernier n'est mentionné que dans le troisième, et sans aucun qualificatif ${ }^{\natural}$. Dans la «Fleur des Histoires», écrite bien plus tard, le roi n'a jamais droit à aucun éloge, il est plutôt représenté comme irrésolu sinon irresponsable ${ }^{62}$. Selon le chroniqueur chypriote Amadi, certes très opposé au seigneur de Korykos, ce dernier, apprenant en 1308 la mort de Héthoum II, se montra très content car il l'avait toujours trahi $i^{63}$. Le tempérament comploteur du prince allait en effet réapparaître au début du XIV siècle à l'occasion de la crise chypriote. Dans un cas (1293) comme dans l'autre (1305) il n'en dit rien dans la «Fleur des Histoires», mais aucun chapitre de ce texte ne permet d'infirmer le schéma ci-dessus : complot contre Héthoum II en 1293, exil en 1294, voyages en Perse et en France, retour en 1298 sous le bref règne de Constantin. Quand Héthoum II revint au pouvoir en 1298, peut-être lui avait-il déjà pardonné. Il n'est pas exclu que la reconciliation ait eu lieu en 1295 à Tabriz, car Héthoum II s'y rendit pour rencontrer Baïdou - et se retrouva d'ailleurs devant Ghazan $^{64}$. Or, nous l'avons vu, il est probable que l'époque ait coïncidé avec le voyage du seigneur de Korykos. De toute façon, en 1298 s'annonçait la grande campagne mongole contre les Mamlouks, à laquelle les deux Héthourn allaient participer. L'heure n'était plus aux querelles internes.

Dulaurier et Pâris, écrivant tous deux en 1869, prétendent que le retour du seigneur de Korykos se fit plus tôt, grâce à la médiation du catholicos Grégoire ${ }^{65}$. On ne voit pas sur quelles sources ils se basent. Aussi difficile à défendre est la théorie de Aguinian $^{66}$ : selon lui, Héthoum, fuyant en 1294 après son complot, aurait été déchu de son titre de seigneur de Korykos, repris par Héthoum II alors libéré de son titre royal, ce qui conduit l'auteur à attribuer à ce dernier les deux chroniques écrites en 1296. Or dans les trois colophons du traité de médecine de 1294 on lit Héthoum seigneur de $\operatorname{Korykos}^{67}$, qui ne peut être Héthoum II cité lui aussi dans le troisième.

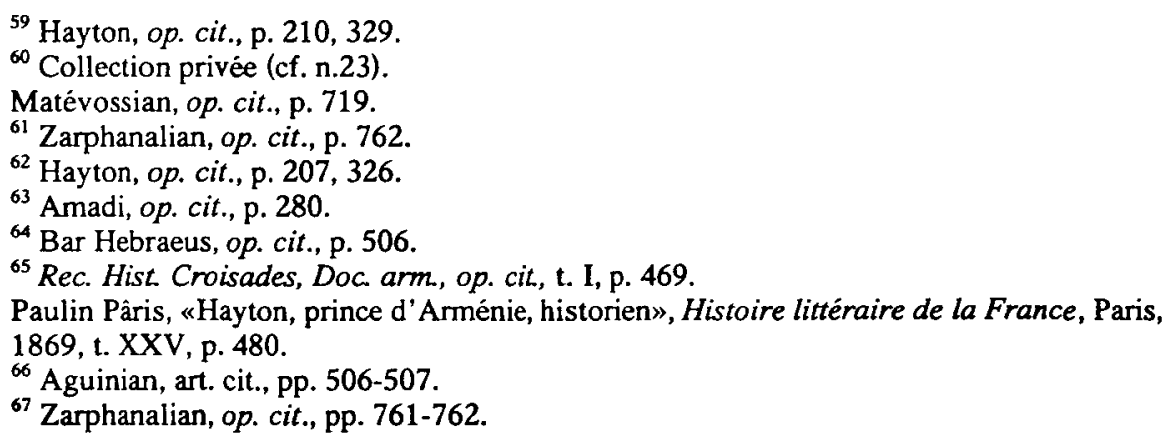


De plus, en 1296, alors qu'il s'apprêtait à reprendre le pouvoir, on voit mal Héthoum II écrivant deux chroniques et les signant toutes deux du nom d'une seigneurie qu'il aurait ainsi reçue. Il semble plus logique de lui attribuer la première - les preuves, on l'a vu, abondent dans le texte —, et de mettre la mention Héthoum seigneur de Korykos sur le compte d'une erreur de copie, tout en laissant à notre «Héthoum l'historien» la propriété de la seconde (jusqu'à nouvel ordre...).

\section{La lutte contre les Mamlouks (1299-1305)}

C'est en 1299 que Ghazan, pourtant récemment converti à l'islam, convoqua le roi d'Arménie et le roi de Géorgie et les autres chrétiens d'Orient' t' $^{6}$ pour sa grande campagne syrienne contre les Mamlouks d'Égypte. Malgré son erreur de date (il donne 1301$)^{69}$ on n'a pas lieu de douter du seigneur de Korykos quand il dit avoir été présent ${ }^{70}$ (il ne parle pas de participation) à la grande victoire remportée à Homs, en décembre 1299, par la coalition christiano-mongole. Il décrit d'ailleurs longuement la bataille, vantant les mérites de Ghazan sans mettre beaucoup en valeur le roi d'Arménie, contre lequel il avait encore du ressentiment. Il ne dit pas un mot de l'éventuelle entrée de Héthoum II à Jérusalem et de son séjour de deux semaines dans la Ville Sainte, que rapporte longuement le dominicain arménien Nersès Balients ${ }^{71}$ (à la date, invraisemblable, de 1302); dans ces conditions, on peut émettre de fortes réserves sur cet «événement» ${ }^{72}$.

Sa présence aux affrontements entre Mongols et Mamlouks au tout début du $\mathrm{XIV}^{\mathrm{e}}$ siècle donne une valeur particulière aux détails fournis par le seigneur de Korykos. Il ne peut bien sûr que déplorer la reprise de la Syrie par les Mamlouks, qui rendit vaine cette première (et dernière) victoire mongole. En 1303, Ghazan voulut reprendre les hostilités, arriva à l'Euphrate et manda le roi d'Arménie ${ }^{73}$ - le seigneur de Korykos ne donne même plus le nom du roi. Le khan ne mena pas cette fois en personne l'armée, et Héthoum décrit encore en témoin le désastre de la coalition et les noyades en masse dans l'Euphrate au cour de la retraite ${ }^{74}$.

Cette présence du seigneur de Korykos aux côtés des Mongols de Ghazan semble avoir alimenté des légendes. C'est ainsi que quelques décennies plus tard Giovanni Villani, dans sa relation de la victoire de 1299 suivie de la conquête de la Terre Sainte, montre le khan désireux d'épouser la plus belle femme du monde. Ses ambassadeurs parcoururent le monde et choisirent la fille du roi d'Arménie. Le roi

\footnotetext{
${ }^{68}$ Hayton, op. cit., p. 191, 316.

${ }^{69}$ Id., p. 193, 317.

${ }^{70}$ Id., p. $195,318$.

${ }^{71}$ Chroniques mineures, op. cit., t. II, p. 185.

Rec. Hist. Croisades, Doc. arm., op. cit., t. I, p. 660.

${ }^{72}$ L. Alichan, Hay-Vened, en arménien, Venise, Saint-Lazare, 1896, p. 89.

M. Aghavnouni, Moines et visiteurs de la Jérusalem arménienne, en arménien, Jérusalem, 1929, pp. 223-225.

Cl. Mutafian, Le royaume arménien de Cilicie, Paris, CNRS, 1993, p. 73.

${ }^{73}$ Hayton, op. cit., p. 200, 321.

${ }^{74}$ Id., p. 203, 324.
} 
envoya sa fille avec son frère, le frère Aiton $^{75}$ (les deux Héthoum deviennent ici deux frères...). Celle-ci accoucha d'un monstre, qui une fois baptisé devint l'être le plus beau et le plus gracieux qu'on ait jamais vu. D'où le baptème de Ghazan et de tout son peuple...

En 1305, c'est en tant que participant que Héthoum de Korykos rapporte la victoire d'Ayas, où les Arméniens s'opposèrent à une invasion mamlouke de la Cilicie: de 7000 Sarrazins, il n'en réchappa que $300^{76}$. C'est là que l'auteur termine son récit historique. Selon son texte, cette victoire a fortifié le royaume et l'autorisait enfin à prendre l'habit de religion ${ }^{77}$ : sur le champ de bataille même, il prit congé du roi et de ses parents, et s'embarqua pour Chypre où il se fit moine prémontré dans l'abbaye de Bellapaïs.

\section{Héthoum de Korykos et les affaires chypriotes (1305-1310)}

La manière idyllique dont le seigneur de Korykos raconte son passage à Chypre après la victoire d'Ayas sur les Mamlouks en 1305 est assez suspecte, et totalement contredite par les chroniques chypriotes. Même si ces dernières exagèrent par haine du personnage, le fond de vérité est indéniable. Depuis 1285 régnait à Chypre le faible roi Henri II. Les liens des Lusignan avec la dynastie régnante d'Arménie, qui remontaient au mariage de Léon $I^{\text {er }}$ avec Sibylle, fille d'Amaury $I^{\text {er }}$ de Chypre, au début du XIII ${ }^{e}$ siècle, reçurent un coup de fouet, naturel vu le besoin de solidarité entre les deux seuls États chrétiens subsistant au Levant. Deux sœurs de Henri II épousèrent des princes arméniens, et en 1293 son frère Amaury seigneur de Tyr prit pour femme, à Sis, Zabel sœur du roi Héthoum $\mathrm{II}^{78}$. Au début du XIV siècle, le roi s'était d'ailleurs de nouveau effacé, donnant la couronne à son neveu Léon III, mineur. Héthoum II restait régent et menait toujours les affaires du royaume. Il donna pour épouse au jeune roi une cousine germaine de celui-ci, fille de Zabel et d'Amaury de Tyr.

À cette époque, le Royaume de Chypre était le théâtre d'importants événements. En 1306, Amaury de Tyr renversa son frère Henri II, prenant le titre de «gouverneur de Chypre» avec l'appui des grands barons de l'île" ${ }^{79}$. Peut-être a-t-il été poussé par sa femme, l'ambitieuse princesse arménienne Zabel, la «dame de Tyr». Il n'est pas exclu non plus que Héthoum de Korykos, moine à Chypre depuis 1305, ait lui aussi trempé dans le complot, ce qui expliquerait la haine que lui vouent les historiens «légitimistes» de Chypre comme Amadi, Florio Bustron ou Machéras. On lit ainsi chez ce dernier : Le seigneur de Korykos (...) avait toujours été opposé au bon roi (Henri II), il s'était enfui d'Arménie à cause de ses machinations contre Sire

\footnotetext{
${ }^{75}$ Croniche di Giovanni..., op. cit., pp. 180-182.

${ }^{76}$ Hayton, op. cit., p. 205, 333.

${ }^{77}$ Ibid.

${ }^{78}$ Id., p. 18, 208, 328.
}

Chroniques mineures, op. cit, t. I, p. 86, t. II, p. 105, 108.

Le bréviaire..., op. cit., p. 101.

Geste des Chiprois, op. cit., p. 315.

${ }^{79}$ Geste des Chiprois, op. cit., p. 316.

Amadi, op. cit., p. 249. 
Hayton, l'héritier d'Arménie, et vint trouver le roi Henri, avec femme et enfants. Le bon roi Henri le reçut et le traita bien. Or, de même qu'il avait semé la discorde entre Hayton roi d'Arménie et son frères Thoros (erreur), il causa ici une brouille entre les deux frères (Henri II et Amaury), au point que le 7 février 1300 (date fausse) on vint arrêter le roi. Le seigneur de Tyr exila son frère le roi Henri et l'envoya en Arménie auprès du roi Hayton son beau-père le roi d'Arménie (il s'agit en fait de Ochine, et de beau-frère), et resta neuf ans (erreur) à la tête du royaume $e^{80}$. Le nombre d'inexactitudes empêche de prendre le texte au pied de la lettre, mais Amadi, plus crédible, confirme que le seigneur de Korykos était fugitif $d u$ roi d'Arménie et qu'il avait conseillé le seigneur de Tyr dans son entreprise ${ }^{81}$.

Il est donc plus que probable que, contrairement à ce qu'il écrit, Héthoum de Korykos se soit retiré à Chypre, de gré ou de force, à cause de ses divergences avec Héthoum II, revenues à la surface à la fin de la parenthèse mongole. On peut même supposer plus. Amaury avait soutenu avec autant d'enthousiasme que le seigneur de Korykos la politique promongole au cours des années précédentes, et les deux princes avaient en commun un tempérament ambitieux et résolu. Il est raisonnable de supposer qu'ils se soient entendus pour monter une vaste machination contre les deux «faibles rois» Henri II et Héthoum II, et que Héthoum de Korykos ait rejoint Chypre en 1305 pour mettre en œuvre, avec son complice, le premier stade du plan, le renversement du roi de Chypre. En tout cas, la situation dans l'île était mûre pour un complot, et en soutenant le coup d'état de l'époux de sa sour il avait certainement des arrière-pensées concernant le royaume arménien. Tout ceci est confirmé par le fait que, quelques mois après son arrivée à Chypre, le seigneur de Tyr envoya monseigneur Hayton, seigneur de Korykos comme ambassadeur auprès du pape (...) pour expliquer la raison de son action (la déposition du roi) et chercher la confirmation de son titre de gouverneur du royaume. Amadi ajoute qu'il était chargé de diffamer le roi Henri ${ }^{82}$, mais qu'il revint en mars 1308 sans avoir obtenu le blanc-seing de Clément $\mathrm{V}$; le pape ne se prononçait pas dans cette affaire avant plus ample information. Héthoum passa donc deux ans en France, et c'est durant cette période que fut composée «La Fleur des Histoires de la terre d'Orient», offerte à Clément $\mathrm{V}$ à Poitiers en août 1307.

Malgré l'échec partiel de sa mission, le seigneur de Korykos était persona grata auprès du pape. Il est d'ailleurs cité avec tous les honneurs dans des lettres écrites par Clément V à Poitiers en février 1308. Il est intéressant de noter que ces lettres ne traitent que de problèmes mineurs et ne laissent en rien transparaitre le rôle de Héthoum comme envoyé d'Amaury; d'ailleurs, quelques jours plus tard, le pape mentionnait explicitement un autre nom, Jean de Lombardie, comme ambassadeur d'Amaury, seigneur de Tyr, gouverneur du royaume de Chypre - ce qui était une reconnaissance de facto du coup d'état ${ }^{83}$. Par contre, quelques mois plus tard, une lettre écrite par l'envoyé spécial du pape trahit explicitement une mission de

${ }^{80}$ Leontios Makhairas, Chronicle, éd. et trad. anglaise Dawkins, Oxford, Clarendon Press, 1932, t. I, pp. 58-59.

${ }^{81}$ Amadi, op. cit., p. 254.

${ }^{82}$ Florio Bustron, Chronique de l'île de Chypre, éd. René de Mas Latrie, Paris, 1886, p. 162. Amadi, op. cit., p. 280.

${ }^{83}$ Regestum Clementi Papae V, Anni secundus et tertius, Rome, 1886, n 2469. 
corruption que le seigneur de Korykos avait reçue d'Amaury ${ }^{84}$. Héthoum était donc probablement plénipotentiaire de ce dernier pour diverses missions où ses capacités diplomatiques étaient particulièrement bienvenues. C'est ainsi qu'Amadi précise qu'avant de rentrer à Chypre il passa par Gènes en tant qu'ambassadeur du seigneur de $T y r^{85}$; on n'a pas de détails sur cette mission. De plus, les Archives nationales conservent la lettre ${ }^{86}$ écrite par le pape à Lusignan le 20 août 1308 et adressée à Philippe le Bel, lui transmettant des renseignements sur les Templiers d'outre-mer et les préparatifs maritimes du Soudan (le sultan mamlouk), contenus dans des lettres que nous avons reçus de nos chers fils, noble homme Amaury, seigneur de Tyr, gouverneur du royaume de Chypre et frère Héthoum parent du roi d'Arménie, seigneur de Korykos - le nom Héthoum est en fait écrit Hanton, d'où la tradition erronée qui prétend que le seigneur de Korykos serait entré en religion sous le nom de «Antoine».

Amadi précise ${ }^{87}$ qu'il revint à Chypre muni d'une lettre destinée à Amaury et concernant les Templiers, alors en voie de liquidation. Là, il apprit ce qui s'était passé en Cilicie en 1307 : dans un guet-apens tendu par le gouverneur mongol, le roi Léon III et le régent Héthoum II avaient été assassinés. Le seigneur de Korykos s'embarqua alors immédiatement pour la Cilicie, ce qui confirme bien que son départ de 1305 était lié à l'animosité entre les deux Héthoum. Le nouveau roi d'Arménie était Ochine, autre frère de Héthoum II.

Pour se débarrasser du roi emprisonné, Amaury de Tyr envoya son épouse Zabel auprès de son frère Ochine afin que celui-ci accepte d'accueillir Henri II en exil en Cilicie. La «dame de Tyr» comptait sur Haiton, seigneur de Korykos, son cousin, un homme de nature déloyale comme on l'a déjà vu, devenu maintenant connétable d'Arménie ${ }^{88}$ selon Amadi. L'ancien fugitif ne tarda donc pas à rentrer en grâce auprès du nouveau roi Ochine, qui lui remit la plus haute dignité du royaume, celle de connétable. Ochine épousa sa fille Zabel, qui mourut en 1310 en donnant naissance au futur roi Léon $\mathrm{IV}^{89}$. L'intervention de Héthoum fut efficace, Ochine accepta, et au début de 1310 le roi prisonnier Henri II fut embarqué à Chypre en direction d'Ayas où l'accueillit, de la part du roi Ochine, le jeune seigneur de Korykos $^{90}$. Il s'agit là sans aucun doute du fils Ochine ${ }^{91}$ de Héthoum, à qui son père

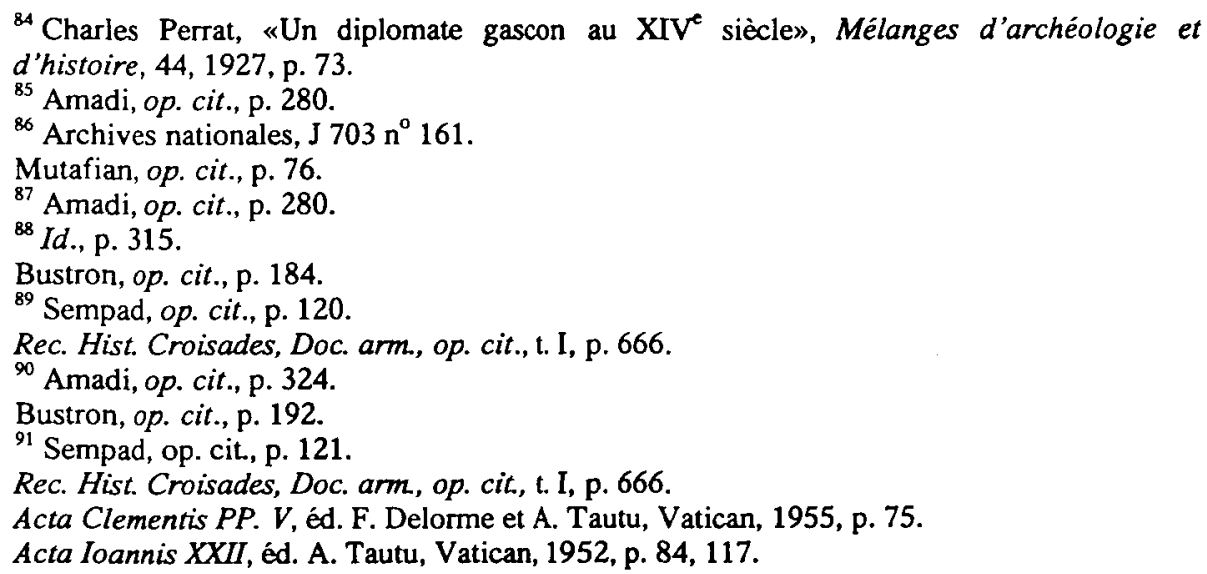


a remis le fief soit à son départ en 1305 , soit à la suite de son élévation au rang de connétable peu après son retour. Ochine allait devenir régent en 1320 quand Léon IV succéda, mineur, à son père Ochine. Les chroniqueurs chypriotes ne se privent pas d'ajouter que Henri II eut une réaction négative en face du jeune seigneur, se rappelant son père qui était méchant et traître ${ }^{92}$. Ajoutons que le nouveau seigneur de Korykos donna sa fille Alice en mariage au roi Léon IV en 1321 alors qu'il était régent ${ }^{93}$. Mal lui en prit: en 1329 le souverain, devenu majeur, le mit à mort ${ }^{94}$. La reine a dû subir le même sort.

Quant à l'affaire chypriote, elle prit fin en 1310 avec l'assassinat d'Amaury ${ }^{95}$. À la suite de nombreuses tractations, Ochine libéra son royal prisonnier qui retrouva le trône de Chypre, en échange de l'autorisation, pour sa sœur Zabel, épouse du défunt usurpateur, de se réfugier en Cilicie avec ses enfants ${ }^{96}$. L'un de ceux-ci, Guy, allait en 1342 devenir Constantin II, premier roi latin d'Arménie.

\section{Héthoum de Korykos et le problème de l'«Union»}

De caractères certes très différents - au roi toujours irrésolu et hésitant s'opposait un seigneur habile, rusé et sûr de lui -, les deux Héthoum avaient beaucoup de points communs. Guerriers valeureux quand il le fallait, ils partageaient la même analyse politique : malgré la conversion de Ghazan à l'islam, l'alliance mongole restait indispensable à la survie du royaume. Mais au fond tous deux avaient un net penchant pour l'Occident latin. Sans que cela entrât en contradiction avec leur goût prononcé pour le pouvoir, ils avaient des tendances monacales: dès la fin du XIII ${ }^{e}$ siècle Héthoum II était devenu fransciscain (sous le nom de frère Jean en hommage au missionnaire Jean de Monte Corvino dont le passage en Cilicie fut sans doute déterminant pour la vocation du roi), alors qu'on a vu Héthoum de Korykos intégrer l'ordre des Prémontrés en 1305.

Cette latinophilie, qui allait se propager dans le royaume quand, peu à peu, la fin de l'alliance mongole ne laissait plus que l'Occident comme secours possible, heurtait une bonne partie du clergé et même de la noblesse, attachée à l'indépendance religieuse traditionnelle des Arméniens. L'opposition la plus forte venait des prestigieux centres monastiques et universitaires de Grande Arménie, directement soumis à la domination mongole et dont la politique n'avait pas de contrainte étatique comme en Cilicie. Elle comptait plusieurs célébrités comme

${ }^{92}$ Amadi, op. cit., p. 324.

Bustron, op. cit., p. 192.

${ }_{93}$ Acta Ioannis XXII, op. cit., p. 84.

Jean Dardel, «Chronique d'Arménie», Rec. Hist. Croisades, Doc. arm., op. cit., t. II, p. 19.

Sempad, op. cit., p. 121.

Rec. Hist. Croisades, Doc. arm, op. cit., t. I, p. 667

${ }^{94}$ Dardel, op. cit., p. 20.

Sempad, op. cit., p. 123.

Rec. Hist. Croisades, Doc. arm, op. cit., t. I, p. 670.

${ }_{95}$ Makhairas, op. cit., pp. 58-59.

Bustron, op. cit., p. 196.

${ }^{96}$ Makhairas, op. cit., pp. 58-59. 
l'archevêque de Sunik (et historien) Étienne Orbélian. La figure de proue de l'opposition à la politique d'Union avec la papauté fut l'Université de Gladzor, fondée vers 1280 et dont la brève existence (à peine plus d'un demi-siècle) fut si riche qu'on l'appela la «seconde Athènes». De 1284 jusqu'à sa mort en 1338, le recteur en fut Essayi de Nitch, dont on a le portrait de son vivant (1318) dû au miniaturiste Thoros de Taron dans un fameux manuscrit, la «Bible de Gladzor»"

Le seigneur de Korykos polémiqua avec Essayi, il lui envoya sa profession de foi, et on possède la réponse du recteur ${ }^{98}$, qu'Alichan ${ }^{99}$ date de 1306 , reprochant à son interlocuteur de se soumettre aux pressions latines et $d$ 'abandonner la tradition arménienne. La lutte entre «latinophiles» et "nationalistes» allait s'exacerber au $\mathrm{XIV}^{e}$ siècle au fur et à mesure de la décadence du royaume, et provoquer des conflits sanglants parmi la noblesse et le clergé arméniens.

La dernière mention qu'on ait de Héthoum de Korykos est encore liée à ses convictions latinophiles, d'ailleurs partagées par tous les rois du début du XIV ${ }^{e}$ siècle : Héthoum II, Léon III et Ochine. Ils n'hésitaient pas à nommer des catholicos de même tendance, et à convoquer des conciles plus ou moins «truqués» pour imposer leurs vues. C'est ainsi que le concile de Sis de 1307 adopta des conclusions tellement favorables à l'Union qu'elles provoquèrent une scission. L'évêque arménien Sarkis de Jérusalem les refusa et, fort de l'approbation du sultan mamlouk, se sépara du catholicos pour fonder le patriarcat arménien de Jérusalem - qui existe encore de nos jours. Le roi Ochine persista dans la voie, et convoqua en 1316 le concile d'Adana. Sur la liste des participants, c'est sans aucun doute notre historien Héthoum qu'il faut voir sous le nom de Hayton Armeniorum dux generalis ${ }^{100}$, avec son rang de connétable (général en chef des armées). Il semble donc que son passage au couvent ait été bien bref, puisqu'il reçut cette charge militaire dès 1308 ou 1309. C'est l'un de ses fils, Constantin seigneur de Lampron, qui lui succéda comme connétable, alors que l'héritier de Korykos était lui aussi présent au concile d'Adana: Oscinus dominus Ghorricusi ${ }^{101}$. Le fait que ce soit Ochine et non son père qu'on trouve comme régent en 1320 suggère que ce dernier était déjà mort, entre 1316 et 1320.

\footnotetext{
${ }^{97}$ Matenadaran (cf. n.32) Ms. 206 fol. $437^{\vee}$.

A. Guevorkian, Miniature arménienne, portrait, Erevan, 1982, $\mathrm{n}^{\circ} 26$.

${ }_{98}$ Matenadaran (cf. n.32) Ms. 579 ff. $316^{\mathrm{v}}-320^{\mathrm{v}}$.

Bibl. mékh. Venise (cf. n.31) Ms. 696/253 ff. 72r sq.

B. Sarghissian, Grand catalogue des manuscrits arméniens..., Venise, Saint-Lazare, 1924, p. 732.

K. Servantzdiants, Thoros aghpar, en arménien, Constantinople, 1884, t. II, p. 395.

M.-A. van den Oudenrijn, Linguae haicanae scriptores o.p., Berne, 1960, pp. 21-22.

${ }^{99}$ Alichan, Sissagan, en arménien, Venise, Saint-Lazare, 1893, p. 132, n.1.

${ }^{100}$ C. Galanus, Conciliationis ecclesiae Armenae cum Romana..., pars prima, Rome, 1650, p. 504,506 .

${ }^{101}$ Id., p. 504.

Sempad, op. cit., p. 130.
} 


\section{«La Fleur des Histoires de la terre d'Orient»}

L'œuvre fondamentale de l'historien Héthoum nous est parvenue en français (médiéval) et en latin. L'incipit du texte français ${ }^{102}$ nous apprend que l'auteur en est frère Héthoum, seigneur de Korykos, cousin germain du roi d'Arménie, et qu'il le compila par le commandement du pape Clément V, en l'an de Notre Seigneur 1307 en la cité de Poitiers. Celui du texte latin ${ }^{103}$ est semblable, les mots cousin germain (qui sont erronés) étant remplacés par consanguin (i.e. parent, ce qui est plus correct). Quant aux explicit, le français ${ }^{104}$ nous dit que le religieux frère Héthoum de l'ordre des Prémontrés, seigneur de Korykos termina son livre sur le passage de la Terre Sainte, et que ce livre fut écrit en français par Nicolas Falcon (certaines leçons précisent de Toul) sous la dictée du frère Héthoum. Nicolas Falcon ajoute qu'il effectua ensuite la traduction latine, datant l'œuvre du mois d'août 1307. L'excipit latin $^{105}$ précise qu'il s'agit du 7 août, et il omet la précision sur le passage de la Terre Sainte.

L'auteur divise lui-même son œuvre en quatre parties. La première est une description des royaumes d'Asie, qui commence par le Cathay ou Chine du Nord et suit un ordre approximatif d'est en ouest. Le chapitre IX est consacré au royaume d'Arménie; il s'agit en fait de la Grande Arménie, dont les habitants ont pris certaines mours des Tartares car ils ont été longtemps sous leur domination. Le chapitre suivant concerne la Géorgie, qui confine au sud avec la Grande Arménie ${ }^{106}$; sa partie orientale, ou Géorgie proprement dite, est sous domination mongole alors que l'occidentale, ou Abkhasie, ne fut jamais soumise à l'empereur d'Asie (i.e. au khan mongol). Le quatorzième et dernier royaume décrit est la Syrie, divisée en quatre parties, autour de Damas, Jérusalem, Antioche et Tarse. La dernière est la Cilicie, à cette époque sous le pouvoir du roi d'Arménie, car depuis que les ennemis de la foi chrétienne ont enlevé cette terre des mains des Grecs, les Arméniens réussirent à recouvrer la Cilicie ${ }^{107}$. Pour certaines parties, l'auteur puise largement dans les autres récits de l'époque, comme Plan Carpin et Marco Polo, mais l'ensemble est un remarquable exposé géographique, précis et bien structuré ${ }^{108}$.

La seconde partie $\mathrm{e}^{109}$ traite brièvement des souverains asiatiques, commençant à Mahomet et à l'expansion arabe, passant ensuite à l'invasion turque de l'Asie Mineure et s'arrêtant à l'écrasement de l'empire du Khwarezm par les Mongols, vers 1230 . Là, rien de bien nouveau.

C'est le livre III, parfois appelé «Histoire des Tartares», qui donne toute sa valeur à l'œuvre de Héthoum ${ }^{110}$. Beaucoup plus développé, il est consacré à l'hisoire des Mongols depuis Gengis Khan jusqu'au début du XIV ${ }^{e}$ siècle et représente,

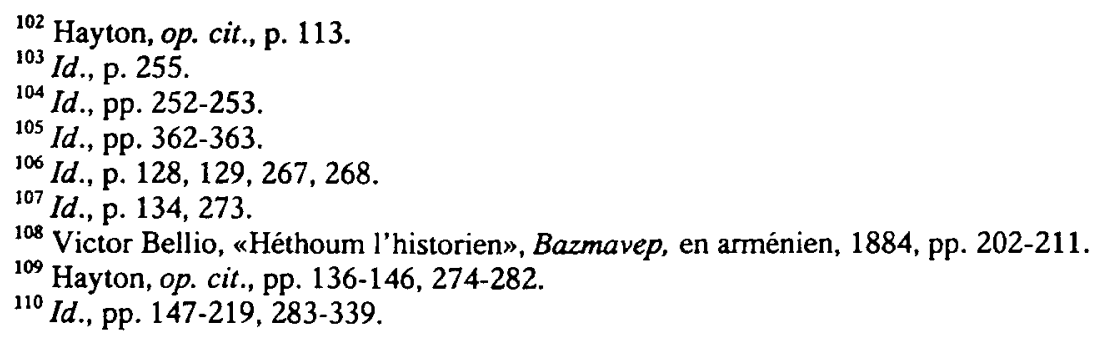


comme on l'a déjà dit, une source contemporaine de tout premier ordre. On a signalé qu'il faut prendre avec précaution l'interprétation des faits quand l'auteur y est directement mêlé. Il convient aussi de tenir compte de l'optimisme parfois exagéré de cet auteur, par exemple quand il parle des sept choses $^{111}$ que le roi Héthoum $I^{\text {er }}$ exigeait du khan Mongka : on a l'impression d'un rapport de forces inversé, le khan ayant accompli les pétitions du roi d'Arménie ${ }^{112}$ avant de... se faire baptiser! Comme allaient plus tard se convertir au christianisme I'ilkhan Houlagou ${ }^{113}$ et le khan Koubilai ${ }^{114}$...

Les trois premières parties ont un contenu qui correspond bien au titre de l'ouvrage. Ce n'est pas le cas de la quatrième, qui n'est autre qu'un projet de nouvelle croisade, certes intéressant mais difficile à justifier dans une histoire de l'Orient. Ajoutant à cette observation de savantes remarques codicologiques, stylistiques ou historiques, Charles Kohler a démontré de manière convaincante ${ }^{115}$ que :

* Héthoum dicta à Falcon les trois premiers livres en français, ils furent aussitôt traduits en latin par ce dernier, et forment «La Fleur des Histoires de la terre d'Orient» proprement dite.

* Le projet de croisade, et lui seul, fut commandé par le pape.

* L'ensemble des quatre livres fut offert à Clément V à Poitiers le 7 août 1305, sous le titre général qui était celui des trois premiers. Kohler prétend de plus, arguments à l'appui, que le projet de croisade fut, contrairement au reste, rédigé d'abord en latin, puis traduit en français et ajouté au texte original de «La Fleur...». C'est possible, sans être aussi certain que ce qui précède.

Le livre IV est donc l'un de ces "passages d'Outre-Mer» qui ont fleuri à partir de la fin du XIII ${ }^{e}$ siècle, dès avant la chute d'Acre et de la Syrie franque en 1291 . Il s'agissait de planifier des expéditions contre l'ennemi principal, le Sultanat mamlouk du Caire, afin de reprendre la Terre Sainte, en tenant compte du fait que subsistaient encore deux États chrétiens, le Royaume latin de Chypre d'abord, puis accessoirement le Royaume arménien de Cilicie, parfois considéré comme moins fiable à cause de l'importance de la faction antilatine. La vogue des "passages» reçut un coup de fouet à la suite de l'enthousiasme provoqué par la victoire - sans lendemain - de la coalition christiano-mongole à Homs en 1299.

Il était naturel que Clément $\mathrm{V}$ profitât du séjour en France du seigneur de Korykos - envoyé, on l'a vu, en 1306 par Amaury gouvemeur de Chypre - pour lui demander son point de vue sur un tel projet : le pape avait rarement dû avoir à sa disposition un pareil connaisseur du Levant. Héthoum commence par présenter l'ennemi, donc l'Égypte et sa puissance, avant de faire quelques rappels historiques (Amaury en Égypte, Saladin, Édouard d'Angleterre, Baibars). C'est au chapitre XI que débute l'exposé des vues de l'auteur ${ }^{16}$, qui, au chapitre XVI, commence $\grave{a}$

\footnotetext{
${ }^{111} I d .$, p. 164, 297.

${ }^{112} I d .$, p. 167, 299.

${ }^{113}$ Id., p. 176, 306.

${ }^{114}$ Id., p. 160, 294.

${ }^{115}$ Id., pp. LVII-LXVII.

${ }^{116} I d$., p. $235,349$.
} 
parler de l'organisation du passage d'outre mer"17. Héthoum préconisait l'organisation préalable d'un «petit passage», mille chevaliers et trois mille soldats sur dix galées débarquant en l'île de Chypre ou au royaume d'Arménie ${ }^{118}$ et, sur le conseil du roi d'Arménie, envoyant tout de suite un message à Carbanda, roi des Tartars - il s'Öldjaïtou, frère et successeur de Ghazan. On reconnaît la patte du seigneur de Korykos: il veut tout de suite que le royaume arménien soit partie prenante, et il reste persua dé qu'il n'y a pas de salut sans les Mongols Je crois fermement, écrit-il, que les Tartares donneraient volontiers en garde aux chrétiens toutes les cités et les terres qu'ils conquèreraient ${ }^{119}$, la chaleur d'été empêchant ces Tartares de demeurer en Syrie. Héthoum insiste sur le fait qu'il connaît assez la volonté des Tartares. Le but de ce "petit passage» serait la conquête de quelques places-fortes en Syrie, pour laquelle cette "avant-garde croisée» pourrait compter sur l'aide des chrétiens de Chypre et de Cilicie. L'auteur demande au pape d'impliquer dans la croisade d'autres chrétiens, les Géorgiens et les Éthiopiens ${ }^{120}$. Pour l'expédition proprement dite, ou «passage général», Héthoum expose trois routes possibles : par l'Afrique du Nord, un choix qu'il laisse à ceux qui connaissent la condition de cette contrée $e^{121}$, par Constantinople, dangereuse à cause de la traversée postérieure de l'Anatolie turque, ou plutôt par mer via Chypre. Au cas où le "petit passage» aurait réussi, on atteindra les ports syriens qui auraient été conquis. En l'absence de tels relais, l'auteur préconise bien sûr le passage de Chypre en Cilicie, où l'armée hivernerait pour ensuite s'emparer d'Antioche et continuer sa descente en Syrie. Il termine en s'appesantissant sur l'alliance indispensable avec les Mongols et en donnant quelques conseils sur la manière de la mettre en pratique. Il voyait bien que cette possibilité allait bientôt disparaître, d'où son insistance à «battre le fer tant qu'il est chaud» : le pape devait se hâter d'organiser l'expédition!

Comme tous les autres, ce projet resta lettre morte. Il semble pourtant que Clément $\mathrm{V}$ ait favorablement accueilli les idées de Héthoum, mais elles furent plus ou moins farouchement combattues par les autres auteurs de projets de "passage», comme Marino Sanuto ou surtout Jacques de Molay. En particulier, l'idée de prendre appui sur le royaume arménien ne reçut que des critiques. D'ailleurs, ne faut-il pas voir là un rêve du seigneur en exil, qui aurait pu ainsi, avec l'«armée de Dieu», reprendre pied sur sa terre natale qui lui était interdite du vivant de son adversaire et homonyme, le roi Héthoum II ? Une belle revanche, qui aurait été à la mesure de ce personnage hors du commun dont la vocation monastique n'était apparemment pas aussi spirituelle qu'on peut le croire.

Claude Mutafian

117 Id., p. 239, 352.

${ }^{118}$ Id., p. $242,355$.

${ }^{119}$ Id., p. $245,357$.

${ }^{120}$ Id. , p. 246, 358.

${ }^{121}$ Id., p. 247, 359. 


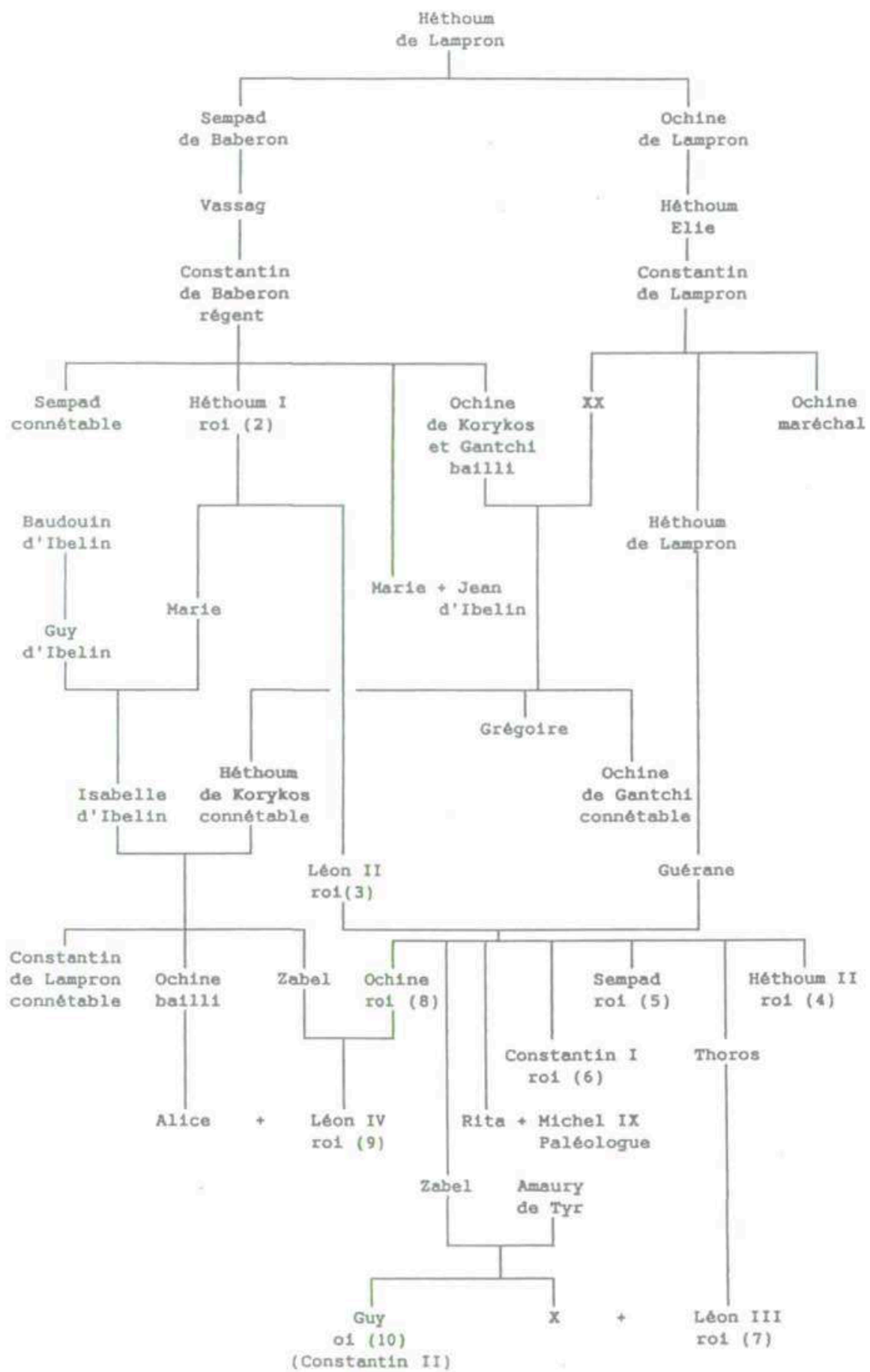

